\title{
The investigation of electronic properties of Ti-based alloys
}

\author{
S.E. Kulkova and D.V. Valujsky \\ Institute of Strength Physics and Materials Science, Siberian Branch of Russian Academy \\ of Sciences, Pr. Akademichesky 2/1, Tomsk 634021, Russia
}

\begin{abstract}
The self-consistent band structure calculations of the ordered and disordered intermetallic titaniumbased alloys were performed using full-potential linearized augmented plane wave (FLAPW) method and Korringa-Khon-Rostoker -coherent potential approximation (KKR-CPA) The electronic structure of the (001) surfaces are also calculated. The alteration of the electronic properties in the three TiMe series (Me=Fe, Co. Ni. $\mathrm{Ru}, \mathrm{Rh}, \mathrm{Pd}, \mathrm{Os}, \mathrm{Ir}, \mathrm{Pt}$ ) are discussed in the dependence of a number of valence electrons. The changes of the electronic structure during martensitic transformation, with disordering and alloying are analyzed. The calculated emission, absorption, electron energy loss spectra and optical properties are found to be in good agreement with experiment.
\end{abstract}

\section{INTRODUCTION}

A number of Ti-based binary alloys crystallize in the cubic B2-stucture at the high temperatures. Some of them become unstable on cooling and the martensite transformation to a stable low-temperatures orthorhombic, monoclinic tetragonal phases accompaing of the «shape memory effects) (SME) are observed. Among the alloys showing SME TiNi is the most prominent example. It is known that shape memory properties of Ti-based alloys are strongly influenced by the type of martensitic transformation (MT), precipitates formed in the matrix and by the addition of the third elements. The phase transitions in the alloys are proceeded by anomalies in various physical properties, which are summarized under the name of premartensitic phenomena. The TiNi crystal structure and properties have been studied in numerous experimental and theoretical works $[1-10]$. Several attempts have been made to explain MT in TiNi by specific features of the electronic structure (ES) of its parent B2-phase. There is no complete agreement between obtained band structure results for TiNi. ES of the monoclinic B19'-phase obtained by LMTO-ASA $[7,9,10]$ and LAPW [8] method are at variance near the Fermi energy. It should be pointed out that the difference in the crystallographic data for B19'-phase [1-4] occurs. The relationship between the instabilities of the B2-phases in the Ti-based alloys and the features of their electronic structure were discussed in the papers [6,8-11]. The role of Ti d-states and their responsibility for the phase instability was stressed in [11]. Despite of numerous attempts to explain the physical nature of MT in Ti-based alloys at the microscopic level there is no complete understanding of the problem. The theoretical description of the influence of alloying on the MT temperature and its sequences is still lacking. Basically ES calculations were carried out using muffin-tin approximation for the crystal potential. The primary objective of the present paper is to study thoroughly the band structure of the three series of B2 Ti-based alloys in the ordered and disordered states and the alteration of the electron characteristics in the different series, during structural transformation and with addition of the third elements. The second purpose is to calculate the surface states of Ti-based alloys.

\section{CALCULATED ASPECTS}

Self-consistent band structure calculation of 9 ordered and disordered B2-TiMe were performed by means of the full-potential linearized augmented plane wave (FLAPW) method [12] with LDA and GGA approximation for exchange-correlation potential. The core states were treated in a fully relativistic fashion. The scalar-relativistic calculations were performed for the valence band states and the $3 \mathrm{~s}-, 3 \mathrm{p}-$ states for $3 \mathrm{~d}$ metals, $4 \mathrm{~s}-, 4 \mathrm{p}-(5 \mathrm{~s}-, 5 \mathrm{p}$-states $)$ for $4 \mathrm{~d}, 5 \mathrm{~d}$ metals respectively. The expansion of the potential and charge density in the muffin-tin spheres was cut off at $\mathrm{l}=10$. Non-spherical contributions to the charge density and potential within MT-spheres were considered up to $I_{\max }=4$. In the interstitial region plane 
waves with reciprocal lattice vectors up to $|G|=14$ were included. The energy spectrum of electrons was calculated at 165 (cubic), 168 (orthorhombic), 128 (monoclinic) k-points in the irreducible part of the Brillouin zone (IP BZ). The MT radii of both Ti and Me atoms were taken to be the same in the series of alloys. The self-consistency is considered to be achieved when the total energy variation from iteration to iteration did not exceed $10^{-5} \mathrm{Ry}$. The crystallographic data from [1-4] were used for B19'-TiNi ES calculations. The lattice parameters of the hypothetical B19-TiNi was extrapolated from the data of Ti-Ni$\mathrm{Cu}$ alloys $(\mathrm{a}=0.4226, \mathrm{~b}=0.2914$ and $\mathrm{c}=0.4526 \mathrm{~nm})$. The surface is simulated by repeated slabs separated in $z$-direction by a vacuum region. The thickness of the vacuum region between the slabs, corresponding to the $8 \mathrm{TiMe}$ layers, was found to be sufficient to avoid interactions of the Ti (Me) atoms. The extensive convergence tests with respect to k-point set and energy cutoff for basis set were performed for a 5- and 7-layer TiNi slab. It was shown that a 5-layer TiNi slab represent a good approximation of the (110)surface but 7-layer TiNi slab must be used for (001)-surface.

\section{RESULTS AND DISCUSSION}

Fig. 1 shows the densities of states (DOS) for ordered and disordered B2-TiMe alloys. Generally speaking, the band structure and DOS for all ordered Ti-based alloys are similar to each other. DOS curves have typical for B2 transition metal alloys two humped structure. The low-energy DOS peak (A) is mainly formed by Me-states and the high-energy one (B) is mainly formed by Ti-states. The analysis of the local partial DOS of TiMe alloys shows that the d-states of Ti and Me are dominated in the DOS structure. For alloys with isoelectronic metals $\mathrm{E}(\mathrm{k})$ and $\mathrm{DOS}$ are almost identical. With increase of a number of the valence electrons (N, el./atom) from 6 to 7 in the series $E_{F}$ moves from deep minimum between two main peaks up towards antibonding $B$-peak. This leads to essential increase of $N\left(E_{F}\right)$. The stability of the $B 2$ phases of TiFe, TiRu and TiOs could be explained by the fact that $E_{F}$ is located in the deep valley separating the «bonding» and «antibonding» states. In TiFe row $N\left(E_{F}\right)$ equal to $5.35,6.79,4.30$ el./(Ry cell) but $N\left(E_{F}\right)$ are $35.38,43.56$ and $36.66 \mathrm{el} . /(\mathrm{Ry}$ cell) in TiNi row. The second trend in the series of alloys is connected with change of DOS structure which is lowered with increase of the charge of the isoelectronic metals and the low energy part of DOS becomes parabolic. The structure of the high-energy DOS part does not change strongly in all Ti-based alloys. As d states split into $t_{2 g}$-like and $e_{g}$-like states in an crystal field the values of the local $t_{2 g}$ and $e_{g}$ contribution to $N\left(E_{F}\right)$ was calculated. It should be pointed out the non-monotone change of the $\mathrm{Me} \mathrm{de} \mathrm{e}_{\mathrm{g}}$-contribution with $\mathrm{N}$ increase for the three series of alloys. In

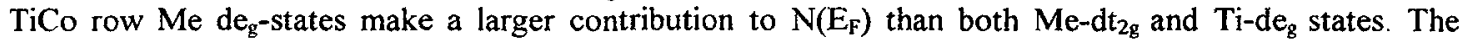
difference between $\mathrm{Me}$ and $\mathrm{Ti} \mathrm{de}_{\mathrm{g}}$-contributions is lowered with increase of the charge of the isoelectronic metals. The increase of $\mathrm{Ti} \mathrm{dt}_{2 \mathrm{~g}}$ contribution to $\mathrm{N}\left(\mathrm{E}_{\mathrm{F}}\right)$ is also

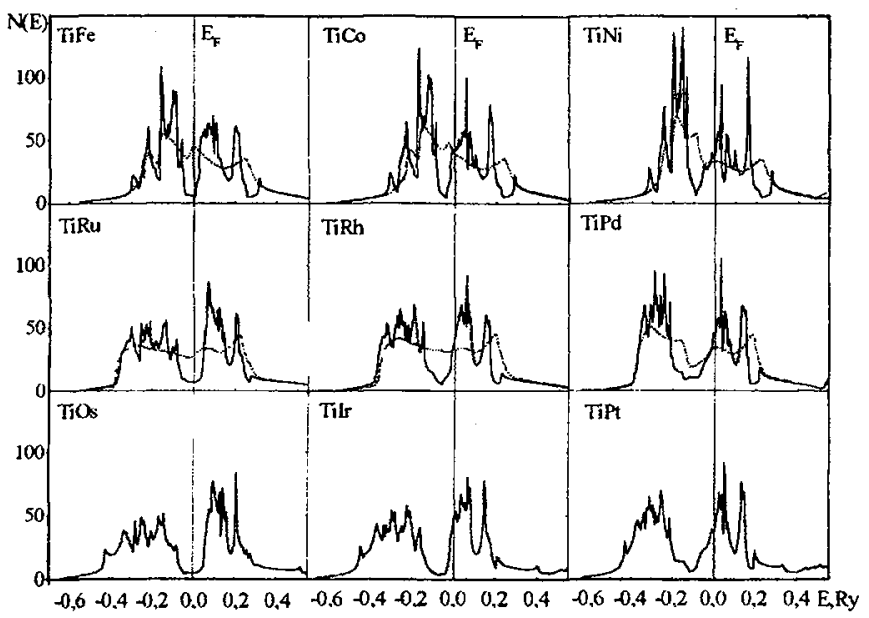

Figure 1 : Total DOS for ordered and disordered Ti-based alloys. observed. The situation coordinally changes in TiNi-TiPd-TiPt set, in which $\mathrm{Ti}$ and $\mathrm{Me} \mathrm{de}_{\mathrm{g}}$ states are of the same order of magnitude but the Ti-contribution is a few higher (9.11 and $8.70 \mathrm{el} . /(\mathrm{Ry}$ atom) in TiNi). The Ti-de $_{g}$ contribution essentially increases in TiPd and TiPt. The results of different authors for the component contributions to $N\left(E_{F}\right)$ for $3 \mathrm{~d}$ TiMe alloys are strongly distinguished, especially for $\mathrm{TiNi}$, but a small exceeds of $\mathrm{Ti} N\left(E_{F}\right)$ is basically marked in the latest papers. Why this ratio of the components contribution to $N\left(E_{F}\right)$ is so important? As mentioned above the 
stability of B2 phases in TiFe-TiRu-TiOs set can be explained using Yamashita criteria and it connected with $E_{F}$ position in deep minimum which separated «bonding» and «antibonding» states. With increase $N$ (from 6 up to 7) $\mathrm{E}_{\mathrm{F}}$ moves up towards peak $\mathrm{B}$ and $\mathrm{Ti}, \mathrm{Me}$ (antibonding» states become progressively filled. In [11] author concluded that $\mathrm{Ti}$ d-states are responsible for phase stability in B2 TiMe alloys because $\mathrm{E}_{\mathrm{F}}$ is dominated by the $\mathrm{Ti}$ d-states. The consideration of the sub-antibonding peak which rises from the valley, where $E_{F}$ is located for alloys with $N$ equal to 6.5 and 7 el shows that it is formed mainly by Me d-states which are as an admixture to Ti-states in this energy region. Thus, the present results and ones from [9] suggest a more complicated picture. The estimation of the charge in this region (from minimum up to $E_{F}$ ) gives $0.68 \mathrm{el}$. of $\mathrm{Co}$ and $1.01 \mathrm{el}$. of $\mathrm{Ni}$ but 0.24 and $0.60 \mathrm{el}$. of $\mathrm{Ti}$ in TiCo and TiNi respectively, whereas in TiPd the contribution of Ti-states exceeds that of $\mathrm{Pd}$. In TiRh and TiPt a number of states of $\mathrm{Ti}$ and $\mathrm{Me}$ are approximately equal but the Ti contribution is less than one of $\mathrm{Rh}$ or $\mathrm{Pt}$. Thus, there is no strong tendency in charge distribution especially in TiNi row. It is possible that the role of metals in the B2-phases stability is very important. Taking into account $\mathrm{Ti}$ - $\mathrm{dt}_{2 \mathrm{~g}}$ contribution in whole $\mathrm{Ti} \mathrm{d}-$ states are dominated at $E_{F}$ in $T i N i$ row. Ti contribution to $N\left(E_{F}\right)$ is virtually twice as high the corresponding one of $\mathrm{Pd}$ (12.31 and 5.37 el./atom $\mathrm{Ry}$ ). In the B19-phase the $\mathrm{Pd}$ contribution of $\mathrm{Pd}$ decreases, while that of $\mathrm{Ti}$ is practically unchanged. On the whole, the decline in $\mathrm{N}\left(\mathrm{E}_{\mathrm{F}}\right)$ during $\mathrm{MT}$ for TiPd and TiNi is estimated to be $14 \%$ and $35 \%$. Pd and Pt are more localized in their spheres than $\mathrm{Ni}$ in B2- and B19-phase. This results in weakening d-d hybridization with Ti d-states. Mixing between Ni (Pd, $\mathrm{Pt}$ ) and $\mathrm{Ti}$ states is reduced in the martensitic phases compared to the austenitic ones. A small change of the bonding especially between Ti atoms in TiNi, TiPd and TiPt can influence on MT in these systems. The decrease of lattice symmetry results to a finer DOS structure. The Fermi level in TiPd, TiPt remains in the vicinity of the local minimum of the high-energy peak in the comparison with the hypothetical B19-TiNi structure (fig.2) in which $E_{\mathrm{F}}$ falls exactly in the sharp peak of DOS that leads to its instability. The addition of $\mathrm{Cu}$ or $\mathrm{Pd}$ on Ni-site stabilizers B19 structure for ternary alloys and $\mathrm{E}_{\mathrm{F}}$ lies in the local minimum like in B19-TiPd. The replacing $\mathrm{Ti}$ by $\mathrm{Zr}$ or Hf does not change essentially DOS structure in the comparison with binary B 19'-TiNi (fig.2). Note that ES results obtained for martensitic monoclinic TiNi in [7-10] are at variance near $E_{F}$. According to Bihlmayer [8] DOS of B19'-TiNi is slightly different from DOS for B2phase. ES of B 19'-TiNi looks quite similar in LMTO $[7,9,10]$ and LAPW [8] calculations in the low energy region but the difference can be detected near $E_{F}$. The four ES calculations of B19'-TiNi were performed with the lattice parameters from [1-4]. These lattice parameters are not much different from one investigator to another. However, the atomic positions show marked distinctions. Submitted in $[3,4]$ the unit cells for monoclinic martensite crystal structure the most precisely describe the results of diffraction experiments. For the variants 1,2 (fig. $3 b, c$ ) the calculated $N\left(E_{F}\right)$ is practically the same or exceeds that for B2-TiNi, which argues against the structural transformation. Of interest is the situation for the third
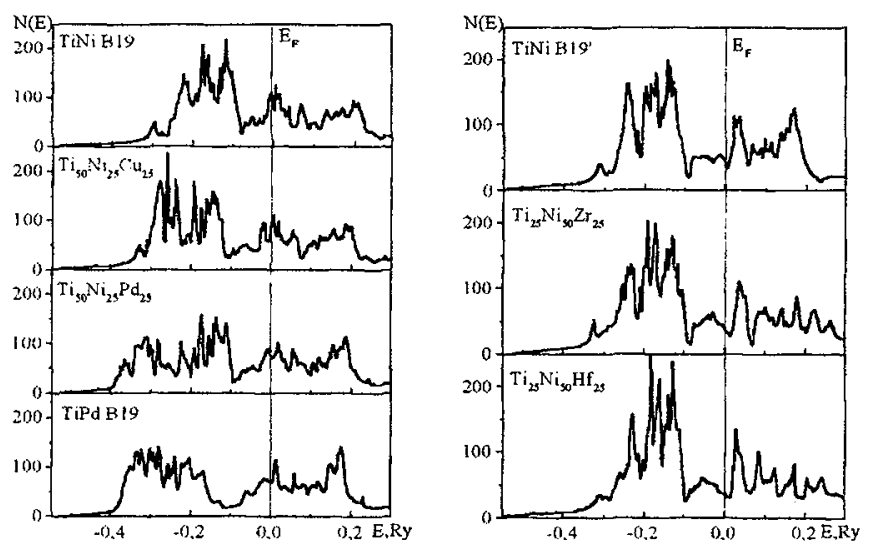
variant. The DOS curve is almost the same as that given in [8]. For the fourth variant DOS curve looks like that obtained by LMTO method in $[7,9,10]$. Despite of a difference between DOS near $E_{F}$ calculated by means of FLAPW and LMTO methods using the parameters from [3], the tendency towards the appearance of states in the energy gap (typical for B2phase) between two groups of bands genericaily related to the components of the alloy, remains in both methods. Thus, the analysis of $N(E)$ curves has shown that for all

Figure 2: Densities of states of ternary TiNi-based alloys. lattice parameters and atomic 
positions in the unit cell the characteristic redistribution of DOS near $E_{F}$ is observed. The change of DOS structure near $E_{F}$ depends essentially on the atoms displacements from their position in the parent B2-phase. The total energy $E_{\text {tot }}$ during MT varies whithin 0.005 Ry for variant 4 (fig.3e). All these features together specify the higher stability of B19'-TiNi at low temperatures as compared to the B2-phase.

It should be pointed out that the DOS changes with disordering (fig. 1) are similar in the two series of alloys. As seen from fig. 1 the total width of DOS in the disordered state is approximately equal to that in the ordered one. The results are quite interesting in that the DOS of TiPd in disordered state looks more similar to that in the ordered state than DOS of other Ti-based alloys. The valley in the middle region is in TiPd DOS structure. The partial DOS of Ti shifts upwards relative to that of $\mathrm{Fe}$ to fill up the energy gap and the peak at $E_{F}$ is appeared in TiFe. As illustrated in fig. 1 the ordered state all Ti-based alloys is highly stable. This confirms by comparison of band energy for two states. The results shown that the possibility to be disordered under special condition in the case of TiPd it is necessary to take into account.

The investigation of the surface properties of shape memory materials is very important for their medical

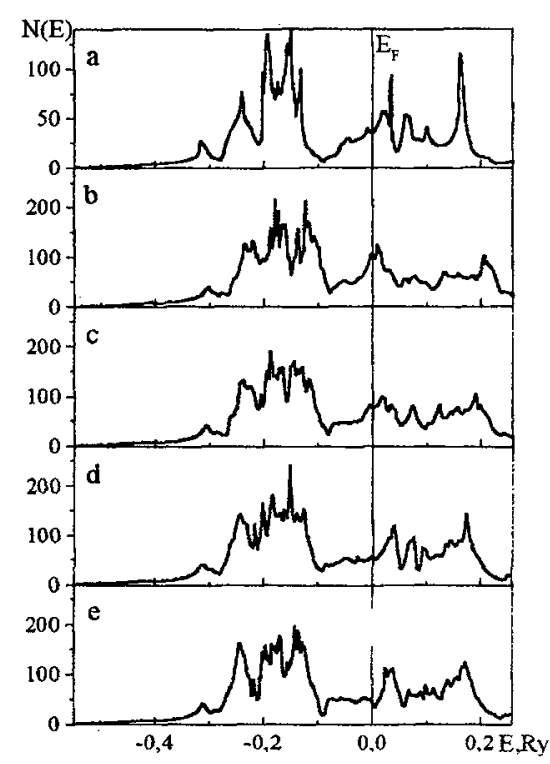

Figure 3: Total DOS of B2-, B19'-TiNi with lattice parameters from papers $[1-4]$. application. It is known that the surface oxide film $\mathrm{TiO}_{2}$ formed in TiNi [13]. In general Ti is good implant materials but $\mathrm{Ni}$ is famous as a hazard materials. Until recently nothing was known about ES TiNi surface. The calculated layer local DOS for 7-layer TiNi(001) films which can be Ti- or Ni-terminated, are shown in fig. 4. The calculation for $3 \mathrm{~d}$ Ti-based alloys shown that 7 layers slab represent a good approximation of the (001) surface and 5 layers slab is found to be sufficient to represent of the (110) surface. In the $3 d-$ TiMe alloys the surface layer is dramatically different from that of the «bulk» $(\mathrm{S}-2)$ layer. The effect of the
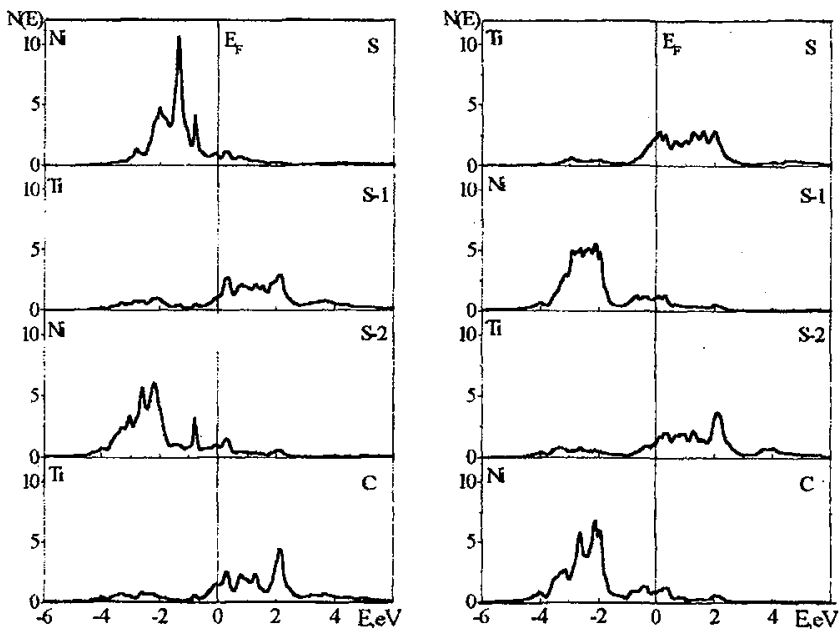

Figure 4: DOS for $\mathrm{Ni}$ - and Ti-terminated 7-layer TiNi(001) films. The symbols $C, S-2, S-1$ and $S$ denote the central, second from center, subsurface and surface layers respectively. presence of surface is essentially decreased in the subsurface layer already. The surface states (SS) at the Ti-terminated surface are located in the vicinity and above $E_{F}$ but in the Ni-terminated surface they are below $\mathrm{E}_{\mathrm{F}}$. For Fe-terminated surface Fe SS shift towards $E_{F}$. Further we found out that the surface layer of $\mathrm{Fe}$ and Co-terminated surface exhibits the magnetic moment which is essentially reduced into the center of films $\left(2.26\right.$ and $\left.0.40 \mu_{\mathrm{B}}\right)$. Ti atoms at the TiNi(001) surface posses a higher reactivity than the $\mathrm{Ni}$ atoms For TiNi(110) surface the ES of surface layer are also different from that of the central layer but the ES of the subsurface layer look like that of central layer. It is known that the high chemical activity of $3 d$ metals is 

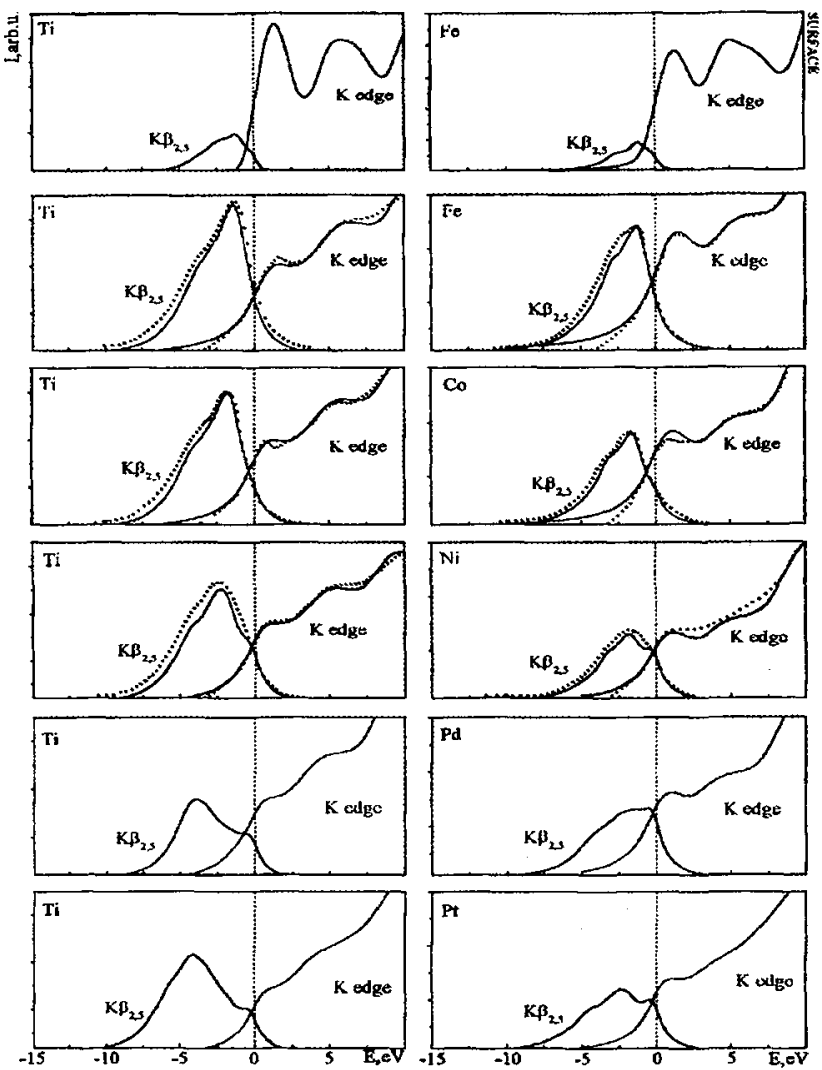

Figure 5: K-emission and absorption spectra for Ti-based alloys. due to by high DOS at $E_{F}$. It follows from obtained results that TiNi surface can not be bioinert. Moreover TiFe surface is not so stable like bulk alloy or TiCo surface.

The obtained ES of Ti-based alloys was used for calculation and interpretation of X-ray emission and absorption $\mathrm{K}$, Lspectra (fig.5). The theoretical X-ray spectra are calculated taking into account the matrix element. The calculated spectra were smeared with a spectrometer function of half-width $0.6 \mathrm{eV}$. The lifetime broadering with a Lorentzian of half-width $1.0 \mathrm{eV}$ was applied to curves. A comparison of the calculated K-spectra with experiment (dotted line) shows a satisfactory agreement in the position and intensity of main peaks. It is thought, that in whole the shape of the spectrum maps a distribution of the valence $d$-states. The basic singularities of $K \beta_{25}$-spectra coincide with the center of the gravity of de $_{g}$ and $\mathrm{dt}_{2 \mathrm{~g}}$-bands. With increase of the charge of the component the atomization of the triplet states occur because the further filling of $d$ band is related to these states. In the comparison with spectra of metals forming $3 \mathrm{~d}$ series of alloys, the intensity of the $\mathrm{Ti}$ spectrum increases in the alloys, but the intensity of the $\mathrm{Fe}-\mathrm{Co}-$ and $\mathrm{Ni}$ - spectra

decreases. The displacement of $E_{F}$ in the region of Ti DOS peak and the filling of the antibonding de $e_{g}-$ states results in the appearance of peculiarity near $\mathrm{E}_{\mathrm{F}}$ in $\mathrm{Ti}$ and $\mathrm{Ni} \mathrm{K} \beta_{25}$-spectrum. This peak becomes more expressed in Ti-spectrum for TiPd and TiPt, in which $\mathrm{Ti} N\left(\mathrm{E}_{\mathrm{F}}\right)$ increases. The low-energy peak in TiPd practically disappears. The maximum of the intensity in the Pd-spectrum near $E_{F}$ does not vary up to -3 $\mathrm{eV}$. The structure of $\mathrm{K}$-spectra in TiFe row differs from considered above and the broad double peak in TiRu and TiOs occurs. The isolation of a selective line of the absorption is observed in all alloys. It is basically expressed for Me-spectra. As the absorption spectra reflect of the states above $\mathrm{E}_{\mathrm{F}}$, the difference in the splitting of d-band into $e_{g}$ and $t_{2 g}$-subbands exhibits in the width of the selective line, which increases in TiFe-TiCo-TiNi set, but it decreases in TiNi row. This behavior agrees well with partial DOS of alloys. In transition metals alloys the $\mathrm{L}_{3}$ spectrum has a rather simple shape without expressed finer structure. Besides it shifts towards the high energy region and is lowered in the comparison with K-spectrum. The calculated L-spectra of alloys and their changes during MT in TiNi row are also in satisfactory agreement with experiment. The electron energy-loss spectra (EELS) for the first series are shown in the fig.6.
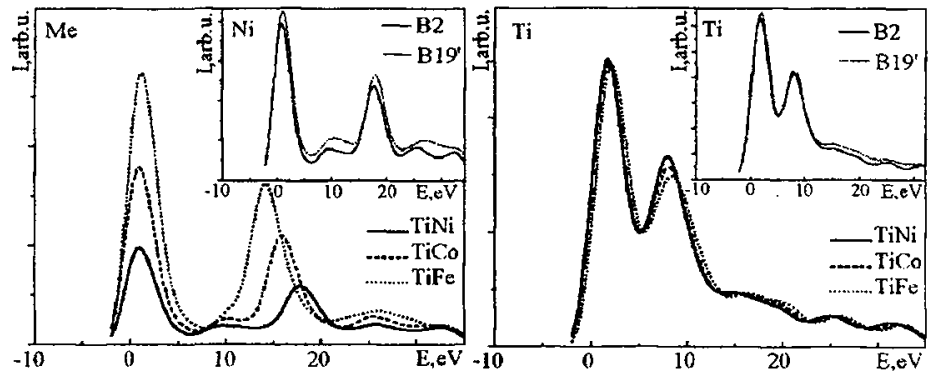

Figure 6: Me and $\mathrm{Ti}_{23}$ edges in TiFe-TiCo-TiNi. 
This spectroscopic technique is rather precisely and its results can be test for ES calculations. Some of the most distinctive features observed in the EELS and X-ray absorption spectra (XAS) of transition metals and their alloys are the characteristic called as a "white line», namely, the sharp maximum of an absorption coefficient. The intensity of a white line reflects changes in the occupancies of the d-states. As it is seen from fig.6. the intensity of the Co white line being twice the intensity of the $\mathrm{Ni}$ line and the more greater increase of intensity of Fe line in TiFe is observed. At the same time the width of the white line sharply decreases in $\mathrm{Fe}-\mathrm{Co}-\mathrm{Ni}$. The calculated Ti-L-spectrum does not reproduce a ratio of the $\mathrm{L}_{3}: \mathrm{L}_{2}$ peaks, observed in an experiment. Within the framework of the one-electron model this ratio should be equal $2: 1$ in the contrary with experiment. The obtained results are shown, that the $\mathrm{Ti}$ band
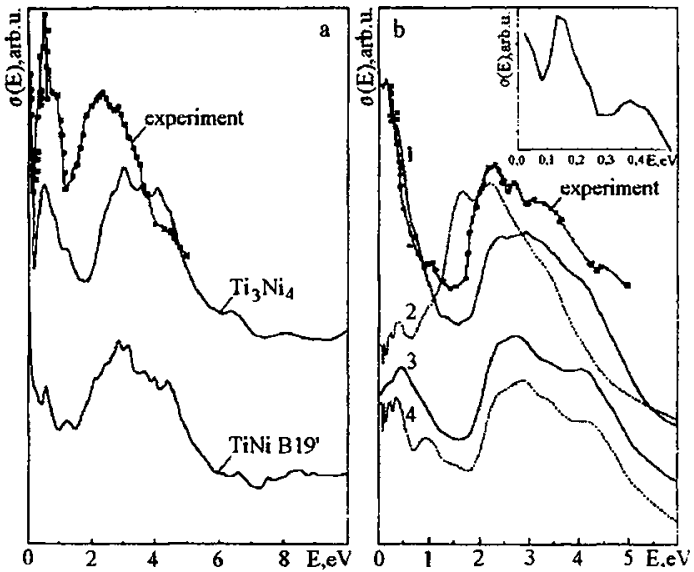

Figure 7: Optical conductivity for $\mathrm{B} 19^{\prime}-\mathrm{TiNi}$ and $\mathrm{Ti}_{3} \mathrm{Ni}_{4}$ (a), curves $1-4$ are $\sigma(E)$ for Ti-and Ni-terminated $(001)$ surface and bulk respectively (b). insignificantly varies in 3d-alloys. $\mathrm{Ni}$ and $\mathrm{Ti}$ bands changes also insignificantly during B2-B 19' MT (the upper inset at the right of fig.6).

It is necessary to point out that the intensive absorption peak at $0.52-0.80 \mathrm{eV} \mathrm{[11]} \mathrm{on} \mathrm{the} \mathrm{optical}$ conductivity curve for B19'-TiNi which was displayed only as peak of very low relative intensity in previous calculation, could be understand taking into account ES change for surface layer. Here we present the result for B2-TiNi. As it is seen from fig. 7 the curve 1 obtained for Ti-terminated film is in good agreement with experiment [11]. Moreover it reproduced well all peaks in the infra-red region which we could not obtain using bulk ES (curve 3,4). It is obvious the change of surface states for B19'-TiNi could be result to the sharp peak at $0.52-0.80 \mathrm{eV}$. Furthermore it is possible the change of the surface composition which depends strongly from the treatment of sample. The calculated optical conductivity for $\mathrm{Ti}_{3} \mathrm{Ni}_{4}$ shows the sharp peak namely in mentioned above region.

So, carried out systematic study of the band structure and electron properties of Ti-based alloys allows to understand the evolution of ES, chemical bond and can be the basis for the analysis of the alloying influence on the sequence of phase transformations.

\section{Acknowledgements}

One of the authors S.E. Kulkova would like to express her deep gratitude to the surgeon A.S. Prosekin for operating her son with application developed by Prof. V. E. Gunter TiNi biomaterial.

\section{References}

1. K. Otsuka, T. Sawamura, K. Shimizu. Phys. Stat. Sol. (a). 5, 457 (1971).

2. R.F. Hehemann, G.D. Sandrock. Scripta metal. 5, 801 (1971).

3. G.M. Michal, R. Sinclair. Acta Cryst. B. 37, 1803 (1981).

4. Y. Kudoh, M. Tokonami, K. Otsuka. Acta metal. 33, 2049 (1985).

5. D.A. Papaconstantopoulos, D.J. Nagel. Int.J. of Quant. Chem. 5, 515 (1971).

6. V.E. Egorushkin, S.E. Kulkova. J.Phys.F: Met.Phys. 12, 2823 (1982).

7. S.E. Kulkova, V.E. Egorushkin. Sol. St. Commun. 77 N9, 667 (1991).

8. G. Bihlmayer, R. Eibler, A. Neckel. J. Phys.: Condens. Matter. 5, 5083 (1993).

9. J.M. Zhang, G.Y. Guo. J. Phys.: Condens. Matter. 7, 6001 (1995).

10. T. Fukuda, T. Kakeshita, et.al. Mat. Sci \& Eng. A273-275, 166 (1999).

11. S.A. Shabalovskaya. Phys. Stat. Sol. (b) 132, 327 (1985).

12. P. Blaha, K. Shwartz, J. Luits. WIEN97. Vienna University of Technology, 1997, 161 P.

13. S.A. Shabalovskaya. J.de Phys.IV. C8-1199 (1995). 